\title{
Auch bei spät Frühgeborenen kann "Lungenreifungs-Spritze" sinnvoll sein
}

Fragestellung: Können Betamethason-Injektionen bei drohender Frühgeburt Komplikationen auch bei Babys verhindern, die in der 34.-36. Schwangerschaftswoche (SSW) zur Welt kommen?

Hintergrund: Späte Frühgeburten haben ein höheres Risiko für das Atemnotssyndrom des Neugeborenen. Weitere Komplikationen können nekrotisierende Enterokolitis, Tachykardie, Gehirnblutungen, Intensivaufenthalte, systemische Infektionen und erhöhte Mortalität sein. Zur Reduktion dieser Risiken und Förderung der Lungenreife wird der Mutter bei drohender Frühgeburt zwischen der 24. und 34. SSW Betamethason injiziert, das ist etabliert [3]. Jedoch besteht auch zwischen der 34. und 36. SSW ein, wenn auch geringer, erhöhtes Risiko für diese Komplikationen [2]. In dieser Zeit kommen 8\% der Neugeborenen zur Welt [1].

Patienten und Methoden: Schwangere in der 34.-36.Schwangerschaftswoche (SSW) mit hoher Wahrscheinlichkeit für eine Frühgeburt wurden in dieser Studie an 17 Zentren in den USA randomisiert mit zwei Injektionen von $12 \mathrm{mg}$ Betamethason intramuskulär im Abstand von 24 h oder Placebo behandelt. Ausgeschlossen wurden Schwangere mit einer absehbaren Geburt innerhalb von 12 Stunden oder mit Kortison vorbehandelte

Schwangere. Als primärer Endpunkt wurde die Not-

Originalie

Gyamfi-Bannerman C, Thom EA, Blackwell SC et al. Network NMFMU. Antenatal Betamethasone for Women at Risk for Late Preterm Delivery. N Engl J Med 2016;374:1311-20. wendigkeit einer respiratorischen Therapie innerhalb von $72 \mathrm{~h}$ nach der Geburt, Beatmungspflichtigkeit oder Todesfall festgelegt. Nachuntersuchungen auf Komplikationen erfolgte 28 Tage nach der Geburt.
Ergebnisse: Zwischen Oktober 2010 und Februar 2015 wurden 1427 aus der Gruppe der mit Betamethason behandelten Schwangeren und 1400 aus der Placebogruppe untersucht. Es gab keine Todesfälle. Das primäre Ereignis trat in der Betamethasongruppe signifikant seltener auf (11,6\% versus $14,4 \%)$. Daraus errechnete sich eine Zahl notwendiger Behandlungen von 35 (95\%-KI 19 bis 259). Auch die Rate an respiratorischen Komplikationen lag in der Therapiegruppe niedriger (8,1\% vs. $12,1 \%)$. An Nebenwirkungen traten neonatale Hypoglykämien in der Therapiegruppe signifikant häufiger auf (24\% vs.15\%).

Schlussfolgerung: Die Autoren schließen auf eine bedeutsame Risikoreduktion für respiratorische Komplikationen durch das Betamethason bei Babys, die in der 34.-36. SSW geboren werden.

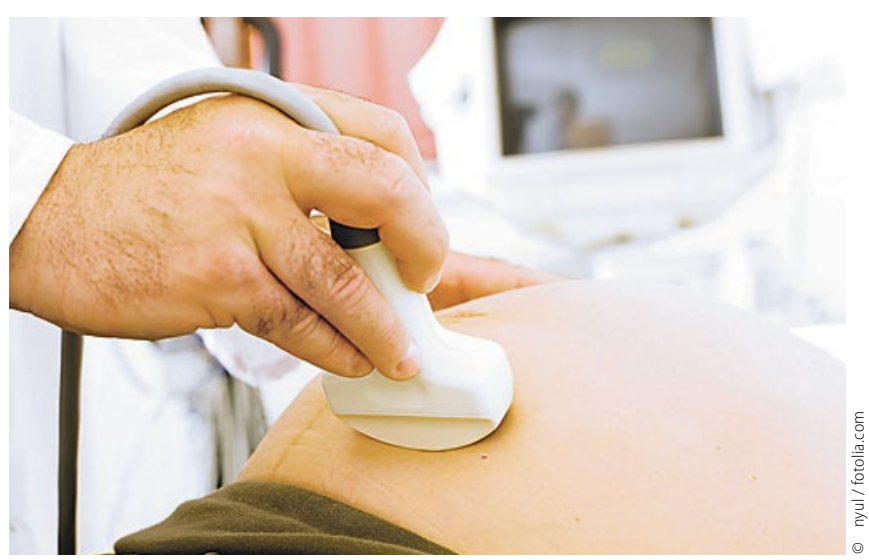

Für die Empfehlung zur Kortisonprophylaxe bei drohender Frühgeburt 4-6 Wochen vor dem errechneten Geburtstermin ist es noch zu früh.

\section{- Kommentar von Prim. Dr. Peter Dovjak}

\section{Erhöhtes Risiko für neonatale Hypoglykämien}

Diese Studie zeigt anhand der Ergebnisse eindrücklich den

3. Roberts D, Dalziel S. Cochrane Database Syst Rev 2006;CD004454

Nutzen einer Behandlung mit Betamethason bei späten Frühgeburten im Hinblick auf eine Reduktion von neonatalen respiratorischen Komplikationen. Jedoch stellt sich angesichts des erhöhten Auftretens von neonatalen Hypoglykämien die Frage nach der Risiko-Nutzen-Bewertung. Die Nachuntersuchungszeit ist dafür zu kurz, eine geplante Nachuntersuchung nach 6 Monaten sollte diese Frage klären, obschon für Klärung eigentlich eine Langzeitnachuntersuchung nötig wäre.

2. Hibbard JU, Wilkins I, Sun L et al. JAMA 2010;304:419-425
}

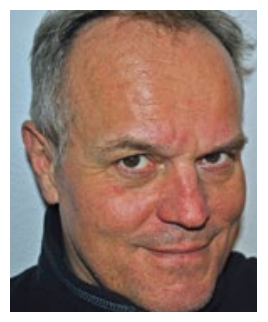

Prim. Dr. Peter Dovjak

Leiter der Akutgeriatrie,

Salzkammergutklinikum

Miller von Aichholzstraße 49

4810 Gmunden

peter.dovjak@gespag.at 